Commun. Korean Math. Soc. 27 (2012), No. 2, pp. 369-375

http://dx.doi.org/10.4134/CKMS.2012.27.2.369

\title{
VOLUME PRESERVING DYNAMICS WITHOUT GENERICITY AND RELATED TOPICS
}

\author{
Jaeyoo Choy, Hahng-Yun Chu, and Min Kyu Kim
}

\begin{abstract}
In this article, we focus on certain dynamic phenomena in volume-preserving manifolds. Let $M$ be a compact manifold with a volume form $\omega$ and $f: M \rightarrow M$ be a diffeomorphism of class $\mathrm{C}^{1}$ that preserves $\omega$. In this paper, we do not assume $f$ is $\mathrm{e}^{1}$-generic. We prove that $f$ satisfies the chain transitivity and we also show that, on $M$, the $\mathrm{C}^{1}$-stable shadowability is equivalent to the hyperbolicity.
\end{abstract}

\section{Introduction}

An important part of the study of dynamical systems is to understand the notions of hyperbolicity, shadowablility and the structures of the attractors and chain recurrent sets. In order to grasp the geometric structure in dynamical systems, the above concepts have played an important role in the field bearing the useful properties.

Our purpose of this paper is to study the hyperbolicity, chain transitivity and the nonwandering property of volume-preserving (or symplectic) $\mathrm{C}^{1}$ dynamics on compact manifolds. What is the distinct aspect of the paper from the existing papers, e.g. $[1,2]$, is that our results here do not assume the genericity of diffeomorphisms in the dynamical system. Since the symplectic diffeomorphisms are automatically volume-preserving, our results in the paper are applicable to the symplectic dynamics as well.

In this paper, we follow the two basic definitions of attractors and chain recurrences due to Conley [3]. Note that even before Conley's definition, other definitions of attractor can be found in several papers (see, for reference, [4]) while the equivalence of those definitions partly remain as conjectures.

Received November 4, 2010; Revised July 25, 2011.

2010 Mathematics Subject Classification. Primary 37C50; Secondary 37C70, 54H20.

Key words and phrases. hyperbolicity, $\mathfrak{C}^{1}$-stable shadowable, chain recurrence, chain transitive.

The first author was supported by Kyungpook National University Research Fund, 2010.

The second author was supported by Basic Science Research Program through the National Research Foundation of Korea(NRF) funded by the Ministry of Education, Science and Technology(2010-0013784). 
Let us look some related results of the dynamics on the compact manifolds. By a theorem of Moriyasu [5], one can see that if the chain recurrent set $C R(f)$ is $\mathcal{C}^{1}$-stably shadowable, then it is hyperbolic (see [7]). In [6], Nakayama and Noda proved that for a minimal flow on a closed orientable 3-manifold, a chain recurrent set of the induced projective flow is the whole projectivized bundle. Recently, it is proved in [7] that if a chain component is $\mathrm{C}^{1}$-stably shadowable, then the chain component is hyperbolic.

In this article, we study the volume-preserving dynamics on compact manifolds; to list some, the chain transitivity (Theorem 2.5), an equivalence between the stable shadowability and the hyperbolicity (Theorem 2.14), etc.

\section{Chain transitivity and shadowing property}

We fix the notations and definitions used throughout the paper. Let $M$ be an $n$-dimensional differentiable manifold with a metric $d$, and $f: M \rightarrow M$ be a $\mathrm{C}^{1}$-diffeomorphism. A volume form $\omega$ on $M$ is a nowhere vanishing $n$-form on $M$. A symplectic form $\omega$ on $M$ is a nowhere degenerate 2 -form on $M$. Here, the non-degeneracy of $\omega$ is the same as its $(n / 2)$-times wedge product $\omega^{\frac{n}{2}}=\omega \wedge \cdots \wedge \omega$ defines a volume form on $M$. Thus, when we say a symplectic form, $n$ is assumed to be even. Integration along the subsets of $M$ defines a Lebesgue measure $m$. Indeed, by the para-compactness of $M$, locally $m$ is written as a product of a $\mathrm{C}^{1}$-function and the standard Lebesgue measure on $\mathbb{R}^{n}$ (via the $\mathcal{C}^{1}$-transition). This clarifies a Lebesgue measurable subset of $M$, a countable union of Lebesgue measurable subsets of $\mathbb{R}^{n}$ (via the $\mathcal{C}^{1}$-transition). Thanks to the well-known theory of Lebesgue measures and Borel measures, one guarantees any compact subset of $M$ is Lebesgue measurable and is of finite measure. By the compactness, the closed balls (with finite radii) are of finite measure, as well.

If one says $f$ preserves $\omega$, this means $f^{*} \omega=\omega$. When $\omega$ is a symplectic form, the $\omega$-preservation implies the volume-preservation. The volume-preservation of $f$ amounts to the measure-preservation. In the case, for a Lebesgue measurable subset $N \subset M$, we have $m(N)=m(f(N))$.

Definition 2.1. A compact $f$-invariant set $\Lambda$ is called hyperbolic if the tangent bundle $T_{\Lambda} M$ has a continuous $D f$-invariant splitting $E \oplus F$ and there exist constants $C>0,0<\lambda<1$ such that $\left\|\left.D f^{n}\right|_{E(x)}\right\| \leq C \lambda^{n}$ and $\left\|\left.D f^{-n}\right|_{F\left(f^{n}(x)\right)}\right\| \leq$ $C \lambda^{n}$ for all $x \in \Lambda$ and $n \geq 0$.

Now, on a compact manifold $M$ with a volume-preserving diffeomorphism, we study chain recurrence sets. And, we prove that if $M$ is $\mathrm{e}^{1}$-stably shadowable, then $M$ is hyperbolic. That is, we can state that the notion of hyperbolicity is expressed in terms of the shadowing property with the conditions for robustness in volume-preserving diffeomorphisms. Therefore, the notions of hyperbolicity and $\mathrm{e}^{1}$-stable shadowablity coincide.

Let us consider chain recurrence theorem and nonwandering theorem on compact manifolds and then show that on the connected compact manifolds 
$M$ with volume-preserving diffeomorphisms, $M$ is chain transitive (thanks to a theorem of Bonatti and Crovisier).

Let $M$ be a compact manifold and $\omega$ be a volume form. Let $\operatorname{Diff}_{\omega}^{1}(M)$ be the set of volume-preserving $\mathcal{C}^{1}$-diffeomorphisms of $M$. Bonatti and et al. [2] proved that for $\mathrm{C}^{1}$-generic diffeomorphisms, the limit set, the non-wandering set and the chain recurrence set coincide, and that they are the closure of the set of hyperbolic periodic points. Also they showed that (assuming that the manifold is connected) there is a residual subset of $\operatorname{Diff}_{\omega}^{1}(M)$ consisting of transitive diffeomorphisms.

The definitions below need not assume the base space is a manifold. Let $(X, d)$ be a metric space and $f: X \rightarrow X$ be a continuous map.

Definition 2.2. An $\varepsilon$-chain (= $=$-pseudo-orbit) from $x_{0}$ to $x_{n}$ for $f$ is a sequence $x_{0}, x_{1}, \ldots, x_{n}$ with the property that $d\left(f\left(x_{i}\right), x_{i+1}\right)<\varepsilon$ for $0 \leq j<n$. A point $x$ is a chain recurrence point if for every $\varepsilon>0$, there exists an $\varepsilon$-pseudoorbit with the starting and ending points at $x$, that is, there exist $n \in \mathbb{Z}_{+}$ and $x_{0}, x_{1}, \ldots, x_{n} \in X$ such that $x_{0}=x_{n}=x$ and $d\left(f\left(x_{i}\right), x_{i+1}\right)<\varepsilon$ for all $i=0, \ldots, n-1$. The chain recurrence set $C R(f)$ of $f$ is the set of all chain recurrence points, i.e.,

$$
C R(f)=\{p \in X \mid \text { there exists an } \varepsilon \text {-chain from } p \text { to itself, for every } \varepsilon>0\} \text {. }
$$

It is obvious that the chain recurrence set $C R(f)$ is closed. This set was defined by Conley [3]. A point $x$ in $M$ is nonwandering with respect to $f$ if any open neighborhood $U$ of $x$, there exists a positive integer $N$ such that $f^{N}(U) \cap U \neq \varnothing$. The set of all nonwandering points of $f$ is denoted by $N W(f)$. The nonwandering set $N W(f)$ is closed and positively invariant. If, in particular, $f$ is bijective, then $f(N W(f))=N W(f)$. In the compact space, the nonwandering set is nonempty.

Remark 2.3. It is easy to show that for a compact manifold $M$ with a volume form $\omega$, a volume-preserving diffeomorphism $f$ is nonwandering. So we get the equality $M=N W(f)$, i.e., every point of $M$ is nonwandering. Note that the nonwandering set is contained in the chain recurrence set, that is, $N W(f) \subseteq$ $C R(f)$. Using these facts, we directly say that the base manifold $M$ is also chain recurrent.

We use (and have used implicitly) the uniform metric on the space $\operatorname{Diff}_{\omega}^{1}(M)$ of volume-preserving diffeomorphisms, given by $d_{U}(f, g):=\sup _{x \in M} d(f(x)$, $g(x))$. A subset $R$ is called residual if it contains a countable intersection of open and dense subsets. If there exists a residual set $R$ in $\operatorname{Diff}_{\omega}^{1}(M)$ such that any $g$ in $R$ possesses the same property $P$, then we call the property $P$ generic. Such a residual set is called a generic set.

Bonatti and Crovisier [2] proved that, generically, a volume-preserving diffeomorphism is transitive in a compact, connected manifold with a volume form. 
Lemma 2.4 ([2, Theorem 1.3]). Suppose that $M$ is a compact connected manifold with a volume form $\omega$. Then, there exists a residual set $\mathcal{G}_{\omega}$ in $\operatorname{Diff}_{\omega}^{1}(M)$ consisting of transitive diffeomorphisms.

Let $f$ be a diffeomorphism on $M$. An invariant subset $A$ of $M$ is chain transitive if for every two points $p, q$ in $A$ and for every $\varepsilon>0$, there exists an $\varepsilon$-pseudo-orbit with the starting point $p$ and the end point $q$, that is, a finite sequence $p=x_{0}, x_{1}, \ldots, x_{n}=q$ such that $d\left(f\left(x_{i}\right), x_{i+1}\right)<\varepsilon$ for all $i$. The sequence $\left\{x_{0}, x_{1}, \ldots, x_{n}\right\}$ is called an $\varepsilon$-chain in $A$ connecting $p$ and $q$.

Theorem 2.5. Let $M$ be a connected and compact manifold with a volume form and $f$ be a volume-preserving diffeomorphism on $M$. Then $M$ is chain transitive for $f$.

Proof. Firstly we denote the transitive residual set in Lemma 2.4 by $\mathcal{G}_{\omega}$. For every positive real number $\varepsilon$, from the compactness of $M$, there exists a positive real number $\delta$ with $\delta<\frac{\varepsilon}{2}$ such that $d(x, y)<\delta$ implies $d(f(x), f(y))<\frac{\varepsilon}{2}$. From the Baire category theorem, we can pick a volume-preserving mapping $g$ in $\mathcal{G}_{\omega}$ such that $d_{U}(f, g)=\sup _{x \in M} d(f(x), g(x))<\delta$. By the definition of $\mathcal{G}_{\omega}, g$ is transitive, i.e., there is a point $p_{0}$ in $M$ whose orbit closure is the whole manifold $M$. For every point $p, q$ in $M$, since the orbit of $p_{0}$ is dense, there are positive integers $m, n$ such that $g^{m}\left(p_{0}\right) \in B(p, \delta)$ and $g^{n}\left(p_{0}\right) \in B(q, \delta)$. We may assume $n>m$. Now we consider the sequence $\left\{p, g^{m+1}\left(p_{0}\right), g^{m+2}\left(p_{0}\right), \ldots, g^{n-1}\left(p_{0}\right), q\right\}$. Thus, by the uniform continuity of $f$, the above sequence is an $\varepsilon$-chain in $M$ connecting $p$ and $q$. This completes the proof.

Remark 2.6. It is clear that every point of the chain transitive set has the property of chain recurrence. Thus, in the connected case, not using Theorem 2.3 we are also able to say that $M$ is chain recurrent for $f$.

Next we state the case of symplectic manifolds.

Remark 2.7. Let $M$ be a connected and compact manifold and $\omega$ be a symplectic form defined on $M$ and $\operatorname{Symp}_{\omega}^{1}(M)$ the set of symplectic $\mathcal{C}^{1}$-diffeomorphisms of $M$. [1] shows that $\mathfrak{C}^{1}$-generic symplectic diffeomorphisms of a compact manifold are transitive, that is, the transitive symplectic diffeomorphisms form a residual subset $\mathcal{G}$ of $\operatorname{Symp}_{\omega}^{1}(M)$.

The following theorem also generalizes a previous result in [2] assuring the density of transitive diffeomorphisms in $\operatorname{Diff}_{\omega}^{1}(M)$.

Theorem 2.8 ([1, Theorem 1$])$. If $(M, \omega)$ is a compact connected symplectic manifold. The set $G$ of transitive symplectic diffeomorphisms forms a dense $G_{\delta}$-set in $\operatorname{Symp}_{\omega}^{1}(M)$. Moreover, the subset $G^{\prime} \subseteq G$ of diffeomorphisms with the unique homoclinic classes $M$, forms a dense $G_{\delta}$-set in $\operatorname{Symp}_{\omega}^{1}(M)$.

Remark 2.9. By the similar method of the proof of Theorem 2.5, we can say that the compact connected symplectic manifold is also chain transitive. 
Definition 2.10. Let $f$ be a diffeomorphism on a compact manifold $M$. A sequence $\left(x_{i}\right)_{i \in \mathbb{Z}}$ in $M$ is a $\delta$-pseudo-orbit of $f$ if for all $i \in \mathbb{Z}, d\left(x_{i+1}, f\left(x_{i}\right)\right)<\delta$. Given $\epsilon>0$, a pseudo-orbit $\left(x_{i}\right)$ is $\epsilon$-shadowed by an actual orbit $\left(f^{i}(x)\right)_{i \in \mathbb{Z}}$ of $f$ if for all $i \in \mathbb{Z}, d\left(x_{i}, f^{i}(x)\right)<\epsilon$. The diffeomorphism $f$ has the shadowing property if for every $\epsilon>0$, there exists some $\delta>0$ such that every $\delta$-pseudoorbit is $\epsilon$-shadowed by some actual orbit of $f$.

Let $\Lambda \subseteq M$ be a closed $f$-invariant set. We say the subset $\Lambda$ of $M$ is locally maximal in $U$ if there exists a compact neighborhood $U$ of $\Lambda$ such that $\cap_{n \in \mathbb{Z}} f^{n}(U)=\Lambda$.

Definition 2.11. A subset $\Lambda$ of $M$ is $\mathcal{C}^{1}$-stably shadowable if $\Lambda$ is locally maximal in some compact neighborhood $U$ and there exists a ${ }^{{ }^{1}}$-neighborhood $\mathcal{U}(f)$ of $f$ such that for any $g \in \mathcal{U}(f), g \mid \Lambda_{g}$ has the shadowing property where $\Lambda_{g}=\bigcap_{n \in \mathbb{Z}} g^{n}(U)$.

The above set $\Lambda_{g}$ is called the continuation of $\Lambda$. Specially, if $\Lambda=M$, we call simply $f$ is $\mathrm{C}^{1}$-stably shadowable.

Let $p, q$ be points in $M$. Then we can consider an equivalence relation $R$ on $C R(f)$ as follows. $p R q$ means, for every $\varepsilon>0$, there exist both an $\varepsilon$-chain from $p$ to $q$ and an $\varepsilon$-chain from $q$ to $p$. Then we call the equivalence classes the chain recurrence classes or the chain transitive components of $f$, simply call the chain components. The components are compact invariant sets and cannot be decomposed into two disjoint compact invariant sets. Denote $C_{f}(p)$ the chain component of $f$ that contains $p$. It is natural that $C_{f}(p)$ is $\mathcal{C}^{1}$-stably shadowable if there is a $\mathcal{C}^{1}$-neighborhood $\mathcal{U}$ of $f$ such that for every $g \in \mathcal{U}$, $C_{g}\left(p_{g}\right)$ has the shadowing property, where $p_{g}$ is the continuation of $p$.

Proposition 2.12. Let $M$ be a compact manifold with a volume form $\omega$ and $f$ be a volume-preserving diffeomorphism on $M$. Then, $M$ is $\mathrm{C}^{1}$-stably shadowable if and only if $M$ is hyperbolic.

Proof. From Remark 2.3, the volume preserving manifold $M$ is chain recurrent, that is, $M=C R(f)$. It is inferred from [7] that $C R(f)$ is $\mathrm{C}^{1}$-stably shadowable if and only if $C R(f)$ is hyperbolic. By the above results, the proof is completed.

The following lemma by Wen et al. states about the local hyperbolicity of each chain component and it is much useful to prove Theorem 2.14 which touches to the global hyperbolicity.

Lemma 2.13 ([7]). Let $p$ be a hyperbolic periodic point of $f$. If $C_{f}(p)$ is $\mathrm{e}^{1}$-stably shadowable, then $C_{f}(p)$ is hyperbolic.

Using the chain transitivity of the volume-preserving dynamics, in the next theorem, we can say that the local hyperbolicity for each chain component is equivalent to the global hyperbolicity for the whole manifold $M$. 
Theorem 2.14. Let $M$ be a compact manifold with a volume form $\omega$ and $f$ be a volume-preserving diffeomorphism on $M$. Assume that every chain component has a hyperbolic periodic point. If every chain component is $\mathrm{C}^{1}$-stably shadowable, then $M$ is hyperbolic. Furthermore, the converse is also true.

Proof. Since the number of connected components of $M$ is finite and the phase space $M$ is nonwandering for $f$, there exists $n \in \mathbb{Z}_{>0}$ such that $f^{n}$ maps each component to itself. That is, the restriction of $f^{n}$ to any connected component is also a volume-preserving diffeomorphism on the component. From Theorem 2.5, the connected component of compact manifold $M$ is chain transitive for $f^{n}$. By the invariance, the connected component becomes a chain component for $f^{n}$. Note that every chain component of $f^{n}$ is included in some chain component of $f$. Thus we can also say that every chain component of $f$ is some finitely many union of connected components of $M$. Then every chain component of $f$ is clopen (=closed and open) in $M$. By the assumption of this theorem and Lemma 2.13, the chain component is also hyperbolic with respect to $f$. Since $M$ is chain recurrent, it consists of finite union of some clopen chain components which are hyperbolic. Then we conclude the first implication. The converse follows directly from the definitions.

In general, the number of the chain components of a compact manifold is infinite. However, in the volume-preserving case, we can obtain a lot of information for the number of the components as follows.

Corollary 2.15. Let $M$ be a compact manifold with a volume form $\omega$ and $f$ be a volume-preserving diffeomorphism on $M$. Assume that every chain component has a hyperbolic periodic point, then the number of the chain components of $M$ is at most finite. More precisely, $M$ is decomposed into finitely many chain transitive components and every component is also composed of finitely many connected components.

Proof. This directly follows from the proof of Theorem 2.14.

Acknowledgement. The authors are deeply grateful to the referees whose remarks helped to improve the manuscript.

\section{References}

[1] M. Arnaud, C. Bonatti, and S. Crovisier, Dynamiques symplectiques génériques, Ergodic Theory Dynam. Systems 25 (2005), no. 5, 1401-1436.

[2] C. Bonatti and S. Crovisier, Récurrence et généricité, Invent. Math. 158 (2004), no. 1, 33-104.

[3] C. Conley, Isolated Invariant Sets and the Morse Index, C.B.M.S. Regional Lect. 38, A.M.S., 1978.

[4] J. Milnor, On the concept of attractor, Comm. Math. Phys. 99 (1985), no. 2, 177-195.

[5] K. Moriyasu, The topological stability of diffeomorphisms, Nagoya Math. J. 123 (1991), 91-102. 
[6] H. Nakayama and T. Noda, Minimal sets and chain recurrent sets of projective flows induced from minimal flows on 3-manifolds, Discrete Contin. Dyn. Syst. 12 (2005), no. 4, 629-638.

[7] X. Wen, S. Gan, and L. Wen, $C^{1}$-stably shadowable chain components are hyperbolic, J. Differential Equations 246 (2009), no. 1, 340-357.

\section{JAEYOO CHOY}

Department of Mathematics

Kyungpook National University

DAEGU 702-701, Korea

E-mail address: choy@knu.ac.kr

HAHNG-Yun Chu

Department of Mathematics

Chungnam National University

DAEJEON 305-764, Korea

E-mail address: hychu@cnu.ac.kr

Min KyU Kim

Department of Mathematics Education

Gyeongin National University of Education

INCHEON 407-753, KOREA

E-mail address: mkkim@kias.re.kr 